
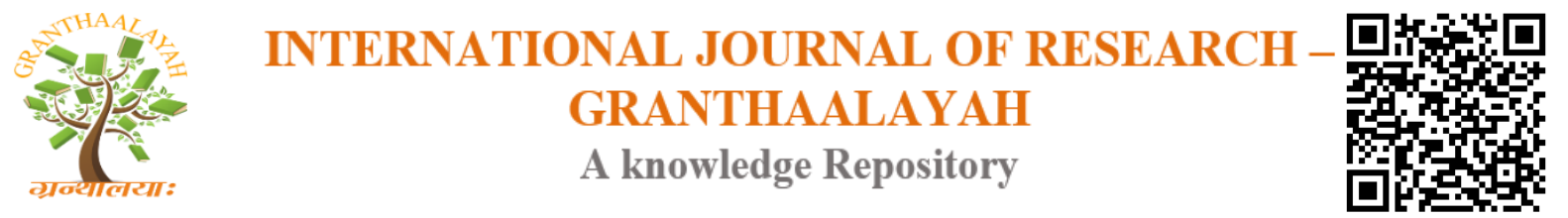

Science

\title{
THE SHEPHERDS HOOK PHENOMENON PATTERN OF HAIR ROOTS A DEMONSTRATION OF COMPARATIVE BIOLECTROMAGNETISM BETWEEN HUMAN HAIRS AND MOUSE WHISKERS BY MEANS OF THE PHOTOELECTRIC EFFECT
}

\author{
Abraham A. Embi Bs *1
}

\begin{abstract}
In this manuscript, the phenomena herein reported have been observed in some of prior publications by this author. This manuscript is a compilation of cross-species similarities on the biomagnetic fields (BMFs) emitted by the human hair and mouse vibrissa follicles. The introduction of a novel optical microscopy technique designed to detect BMFs in plant and animal tissue has allowed researchers to publish interesting and unique findings. They range from hair follicles BMFs penetrating glass barriers to the biomagnetic effect on crystals accretion of fronted hair follicles. Both rodents (whiskers) and human (hair follicles) show similarities regarding spontaneous BMFs expressed as light rays with one sided greater BMF activity. The technique used as a base the iron staining property of Prussian Blue Stain (PBS) mixed with very fine iron particles $2000 \mathrm{~nm}$ in diameter (for magnetic attraction). For this manuscript, previously published images were re-reviewed and addressed as such in the manuscript. Both PBS mixed with the diamagnetic Potassium Ferrocyanide and PBS mixed with the paramagnetic Potassium Ferricyanide were alternatively chosen in those experiments. During evaporation, the hair shaft and follicle, due to their intrinsic diamagnetism, repels the crystals of diamagnetic PBS and attracts the paramagnetic ones. Experiments are presented consistently demonstrating a unique pattern observed of BMFs skewed towards or along one side of the human hair shaft and follicle of humans and rodent vibrissa whiskers.

This is attributed to "The Photoelectric Effect" discovered in 1887 by the German physicist Heinrich Rudolf Hertz.
\end{abstract}

Keywords: Hair Follicles; Rodent Whiskers; Comparative Bioelectromagnetism; Shepherds Hook; Photoelectric Effect.

Cite This Article: Abraham A. Embi Bs. (2018). "THE SHEPHERDS HOOK PHENOMENON PATTERN OF HAIR ROOTS A DEMONSTRATION OF COMPARATIVE BIOLECTROMAGNETISM BETWEEN HUMAN HAIRS AND MOUSE WHISKERS BY MEANS OF THE PHOTOELECTRIC EFFECT." International Journal of Research - Granthaalayah, 6(7), 317-326. https://doi.org/10.29121/granthaalayah.v6.i7.2018.1312. 


\section{Introduction}

The purposes of this manuscript are twofold, first to add an additional publication to the nascent field of Comparative Biophysics and second to introduce a unique property observed, the one sided unilateral biomagnetic fields (BMFs) strength displayed by human hairs and rodent vibrissa whiskers.

\section{Brief History of Hair Follicles Biomagnetic Fields Detection}

During the mid part of the $20^{\text {th }}$ century, sophisticated equipment was utilized to detect Biomagnetic Fields (BMFs) in humans. Organs such as the brain and heart were chosen and extensively studied $(1,2,3)$, a seminal publication by D Cohen in 1980 (4) found magnetic fields produced by steady currents in the human body, specifically in the head by stating "Most of the field over the head is produced by electrical sources associated with hair follicles in the scalp". Thirty-five years later in 2015, a breakthrough utilizing a novel tabletop optical microscopy technique to detect BMFs in plants and animal tissue was introduced in a paper entitled Demonstration of Inherent Electromagnetic Energy Emanating from Isolated Human Hairs (5) duplicating the aforementioned findings of $\mathrm{D}$ Cohen et al. This was possible by the introduction of a novel tabletop technique specifically developed for the imaging of electromagnetic energy in plant and animal tissues (6). Since its introduction, numerous publications have validated BMFs emitted by the human hair follicles and rodent vibrissa whiskers. The publications range from the hair follicle biomagnetic penetration through glass barriers (7) to the introduction of BMFs emanating from rodent vibrissa whiskers (8). The cross-species similarities of BMFs between of the human hair shaft and those of the mosquito larvae were also documented (9).

It is the purpose of this manuscript to continue expanding the book of knowledge as it relates to the field of Comparative Biophysics. This manuscript also presents biophysical similarities of hair follicles between two different species, namely Homo Sapiens and Mus Musculus (mouse).

\section{Materials and Methods}

A revision of files from prior papers by this author and others. It encompassed a selection of images depicting the unique property of BMFs displayed by the human hair follicles and mouse whiskers. To aid the reader interpreting the figures some abbreviations are explained:

NOTE: The author suggest the reader to visit the link for "A Novel and Simplified Method for Imaging the Electromagnetic Energy in Plant and Animal Tissues' listed in reference (6). https://www.semanticscholar.org/paper/A-Novel-and-Simplified-Method-for-Imaging-the-inScherlag-Sahoo/f7b00e9192a975e0d84dcfe9287bb706fd9074c0

\section{Solutions Used}

PBS= Prussian Blue Stain.

$\mathrm{Fe} 2=$ Liquid Potassium Ferrocyanide (diamagnetic, repelling magnetic source)

$\mathrm{Fe} 3=$ Liquid Potassium Ferricyanide (paramagnetic, attracted towards magnetic source)

$2 \mathrm{~K}=$ Fine iron particles size 2000 nanometers $(\mathrm{nm})$ in diameter.

Therefore: 


\section{Working Solutions}

PBS Fe2 2K= Prussian Blue stain solution containing Potassium Ferrocyanide and $2000 \mathrm{~nm}$ iron particles. (Diamagnetic).

PBS Fe3 2K= Prussia Blue stain solution containing Potassium Ferricyanide and $2000 \mathrm{~nm}$ iron particles. (Paramagnetic).

\section{Slide Assemblies}

$\mathrm{SSP}=$ Single slide preparation. Follicles placed in center of a $25 \times 75 \times 1 \mathrm{~mm}$ glass slide; and covered by either PBS Fe2 2k or PBS Fe3 3k.

$\mathrm{SDW}=$ Sandwich. Is when material is trapped between two equally sized glass slides.

\section{Results}

The results are presented pictorially in microphotographs and video recordings from a video microscope, downloaded to an Apple McBook Pro Photo App 8.1.2

\section{Rodent Vibrissa Whiskers}

Rodent vibrissa whisker in SDW with PBS Fe2 2K. Image taken 24 hours post mounting and stored in the dark. Depicts lack the absence of crystals accretion in the left top corner. Also evident is the emission of BMFs expressed as a light beam displacing particles. This is attributed to an absence of BMFs attracting the diamagnetic iron laden solution.

\section{Rodent vibrissa whiskers spontaneously discharging light energy and skewedness of Right sided BMFs}

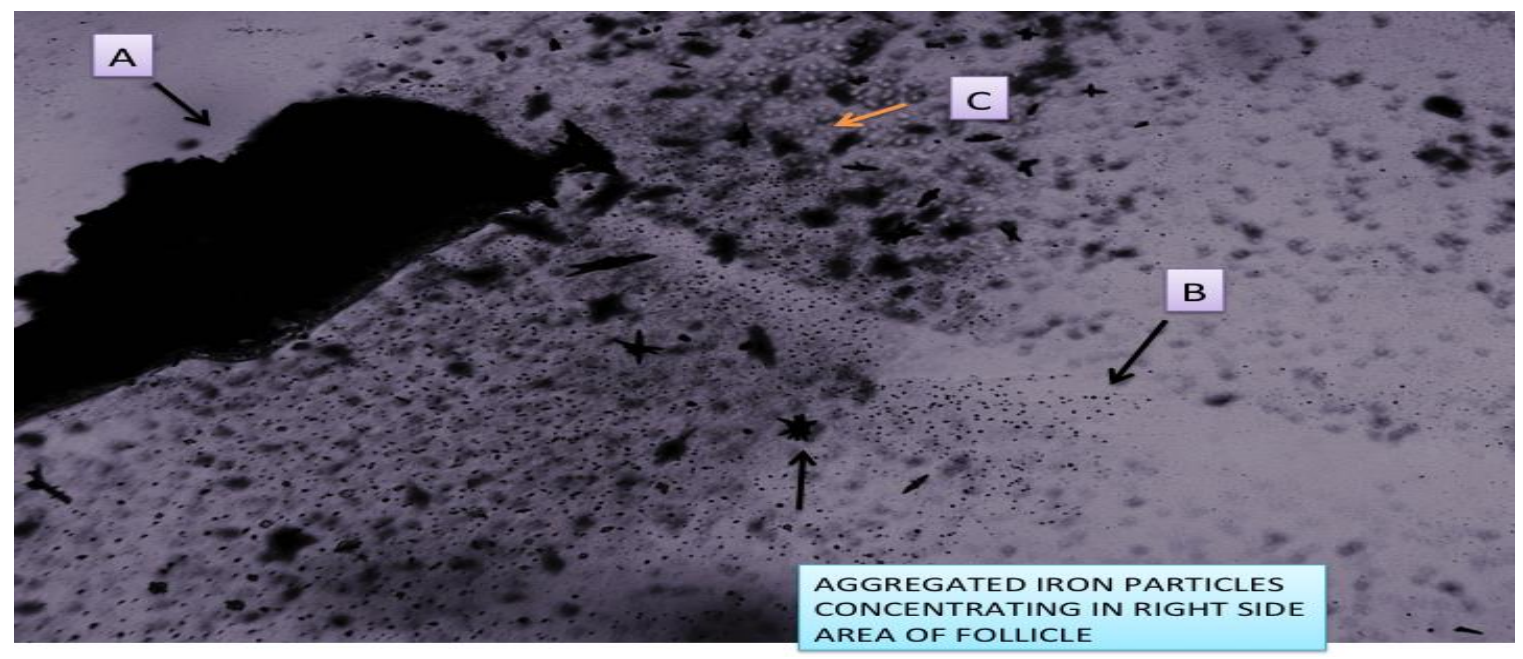

Figure 1: Unpublished image, Example of undisturbed rodent whisker in SDW PBS Fe 2K stored in the dark. A) Follicle B)

Bioelectromagnetic field expressed in the form of light energy. C) Ferrocyanide with Iron crystals partially surrounding follicle. Notice the presence of one sided magnetic activity. Image reproduced from:

Embi AA, Jacobson JI, Sahoo K, Scherlag BJ (2015) Demonstration of Electromagnetic Energy Emanating from Isolated Rodent Whiskers and the Response to Intermittent Vibrations. Journal of Nature and Science, 1(3): e52. 


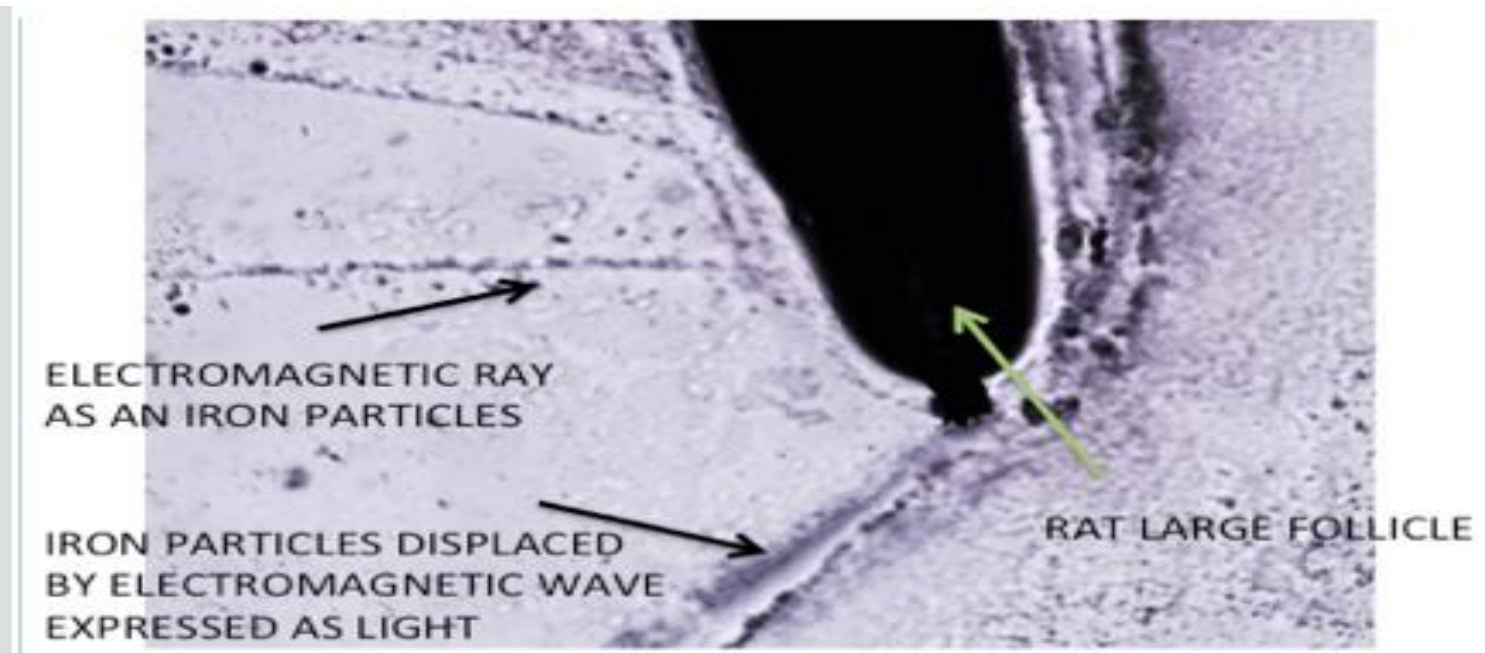

Figure 2: Another large rodent vibrissa whisker unilaterally discharging energy in the form of a light ray. Notice, again the light ray (photons) displacing the PBS stained ferrocyanide + iron

Image reproduced from: particles

Embi AA, Jacobson JI, Sahoo K, Scherlag BJ (2015) Demonstration of Electromagnetic Energy Emanating from Isolated Rodent Whiskers and the Response to Intermittent Vibrations. Journal of Nature and Science, 1(3): e52.

\section{The Human Hair Follicle}

\section{The quintessential Shepherds Hook Image}

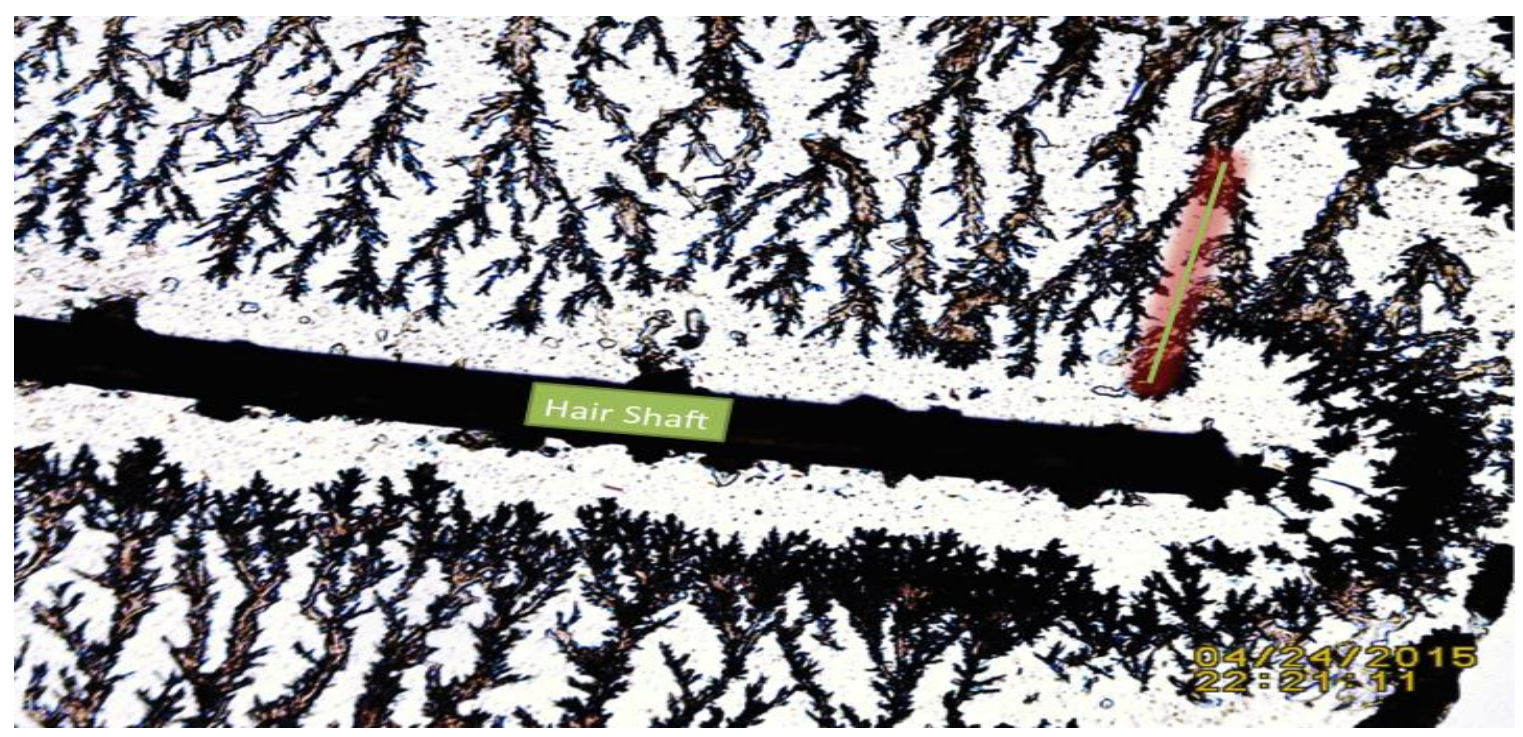

Figure 3: Human hair shaft fragment in SSP PBS FE2 2K after evaporation. Notice Ferrocyanide crystals accretion accentuated in one side of shaft. Highlighted line shows the change in crystals density

Published image in:

Embi AA. Topical In Vivo Liquid Catalase on Scalp and Migraine Headache Cessation in an Adolescent: A Biophysics Based Hypothesis. J Nat Sci, 3(3): e330, 2017. 


\section{Vertical Display of Greater Unilateral BMFs}

In the figure below, the hair follicle is SDW and therefore is at $1 \mathrm{~mm}$ vetical distance from the evaporated PBS Fe2 2K solution. Notice the skewedness of particles (X) towards one side of the fiollicle $(\mathrm{F})$.

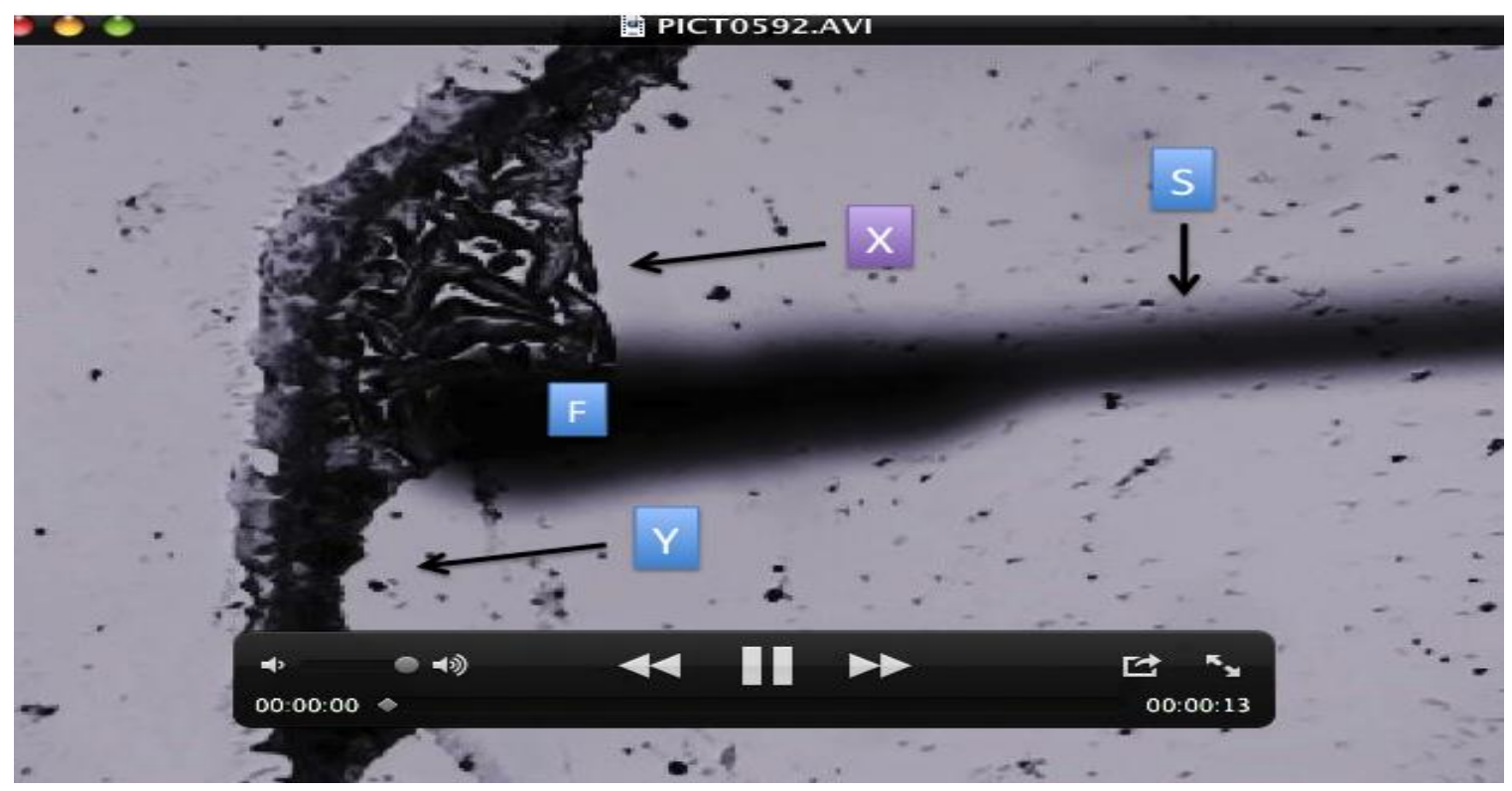

Figure 4: Demonstration of unilateral vertical increased emissions of BMFs of human scalp hair.

The hair follicle mounted in SDW at $1 \mathrm{~mm}$ vertical distance from PBS Fe2 2K evaporated crystals. $\mathrm{F}=$ Out of focus follicle $\mathrm{S}=$ Out of focus hair shaft $\mathrm{X}=$ Unilateral greater accumulation of iron containing crystals $\mathrm{Y}=$ Lesser accumulation of iron containing crystals

\section{Shaft expressing BMFs as Light Energy Displacing Iron Particles}

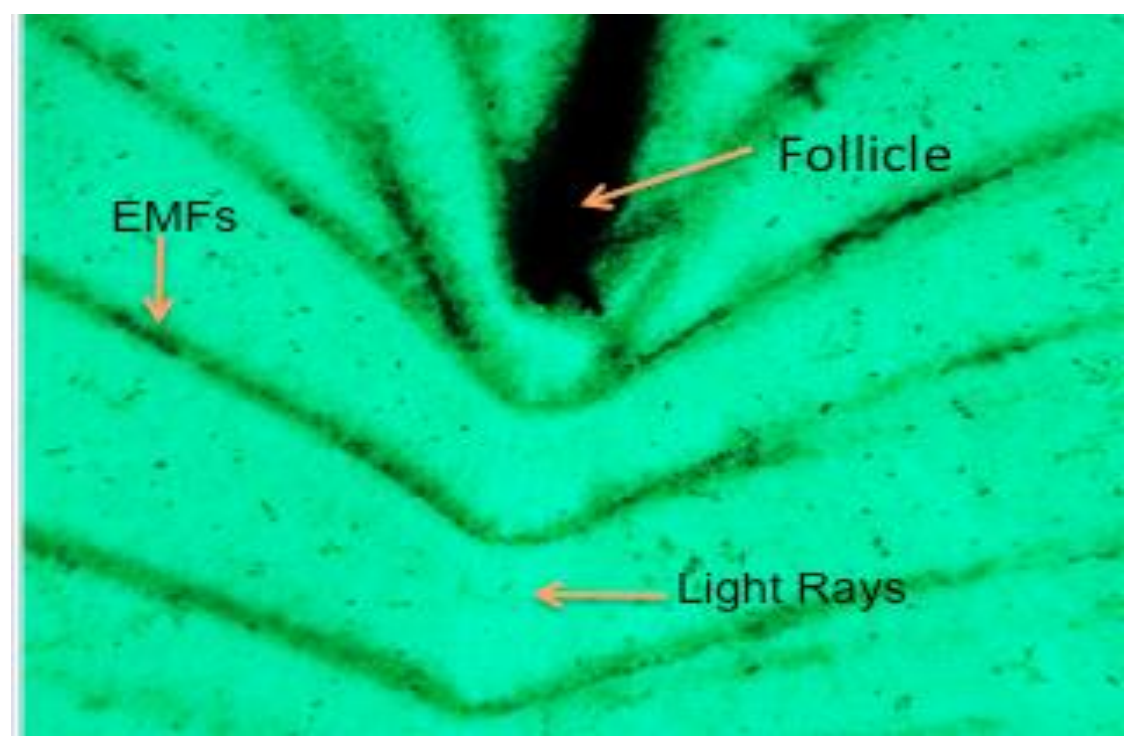

Figure 5: This image shows a human hair follicle in SDW PBS Fe2 2K

The darker lines are Ferrocyanide crystals representing BMFs.

This figure also shows the power of a light ray (photons) displacing particles. 


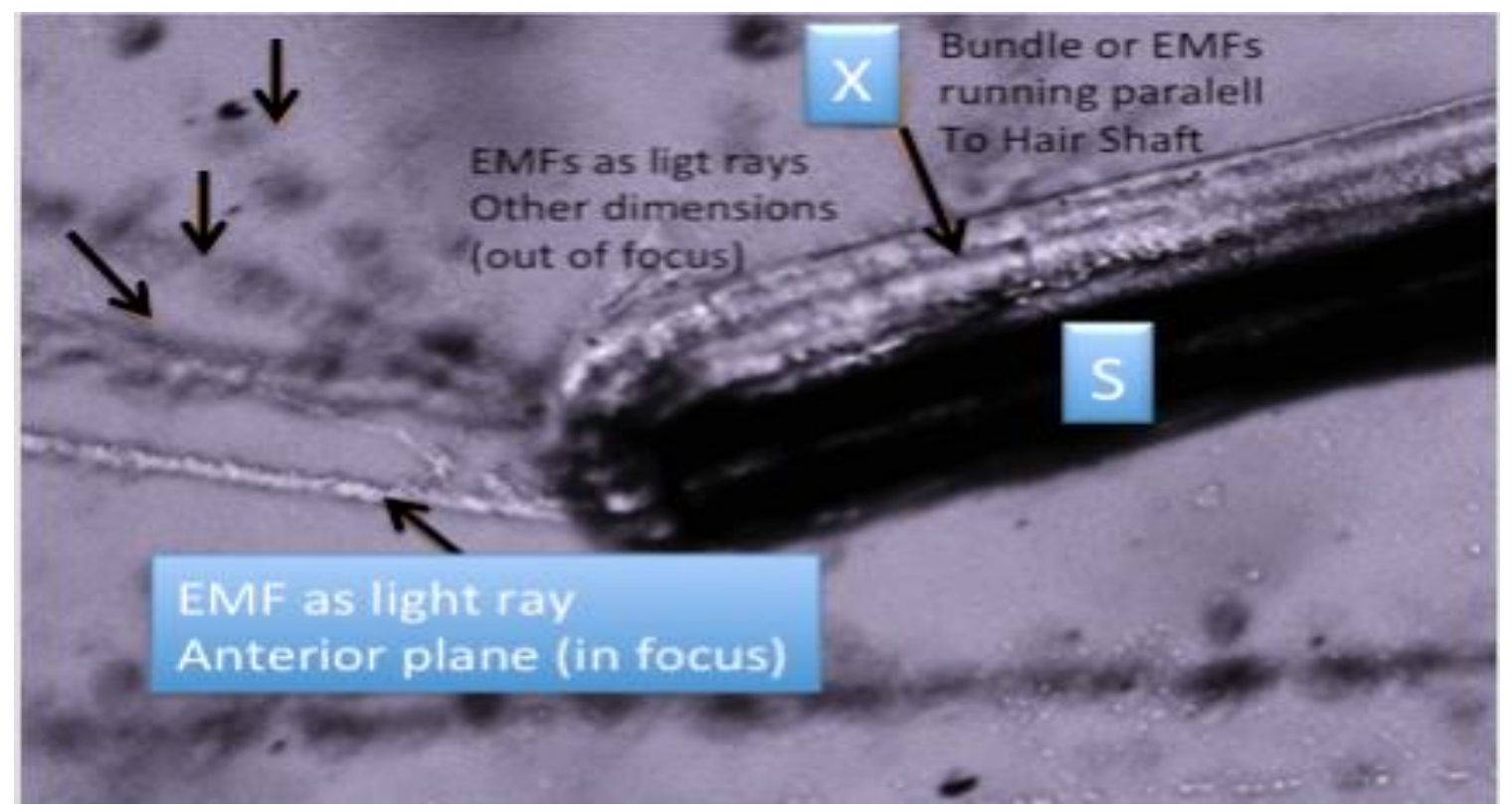

Figure 6: Cut hair shaft end in SDW PBS Fe2 2K after evaporation

This complex figure shows the unilateral BMFs activity in a person during a migraine headache attack. Notice the light energy displayed in different layers. This was seen by changing the microscope depth of focus.

Embi AA. Topical In Vivo Liquid Catalase on Scalp and Migraine Headache Cessation in an Adolescent: A Biophysics Based Hypothesis. J Nat Sci, 3(3): e330, 2017.

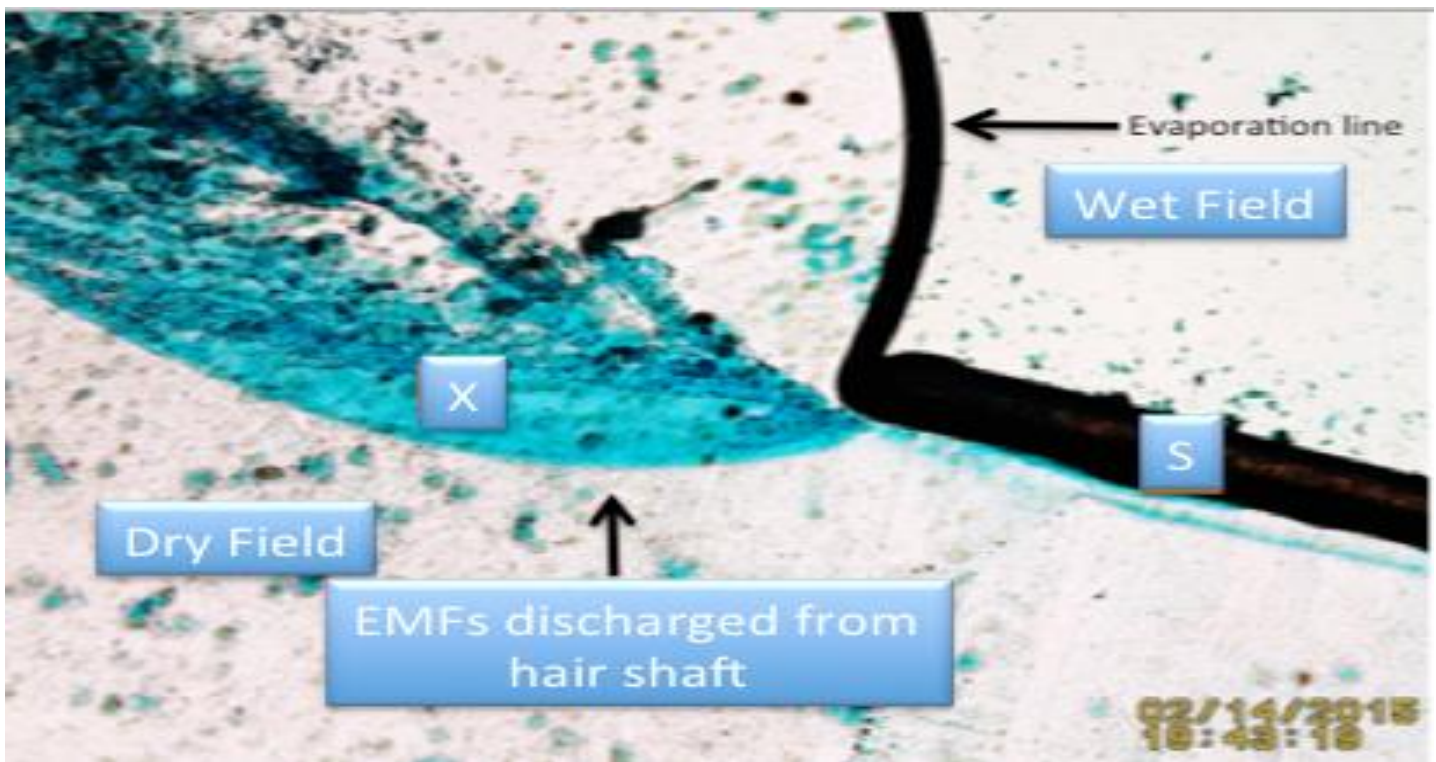

Figure 7: Same specimen similar to Figure 6. This time there is also unilateral energy discharged of the hair shaft. This microphotograph display the crystal accretion as an energy plum. SDW PBS FE2 2K

https://youtu.be/wIt4g21D0Rs 


\section{SSP PBS Fe2 2K solution partially circulating around follicle.}

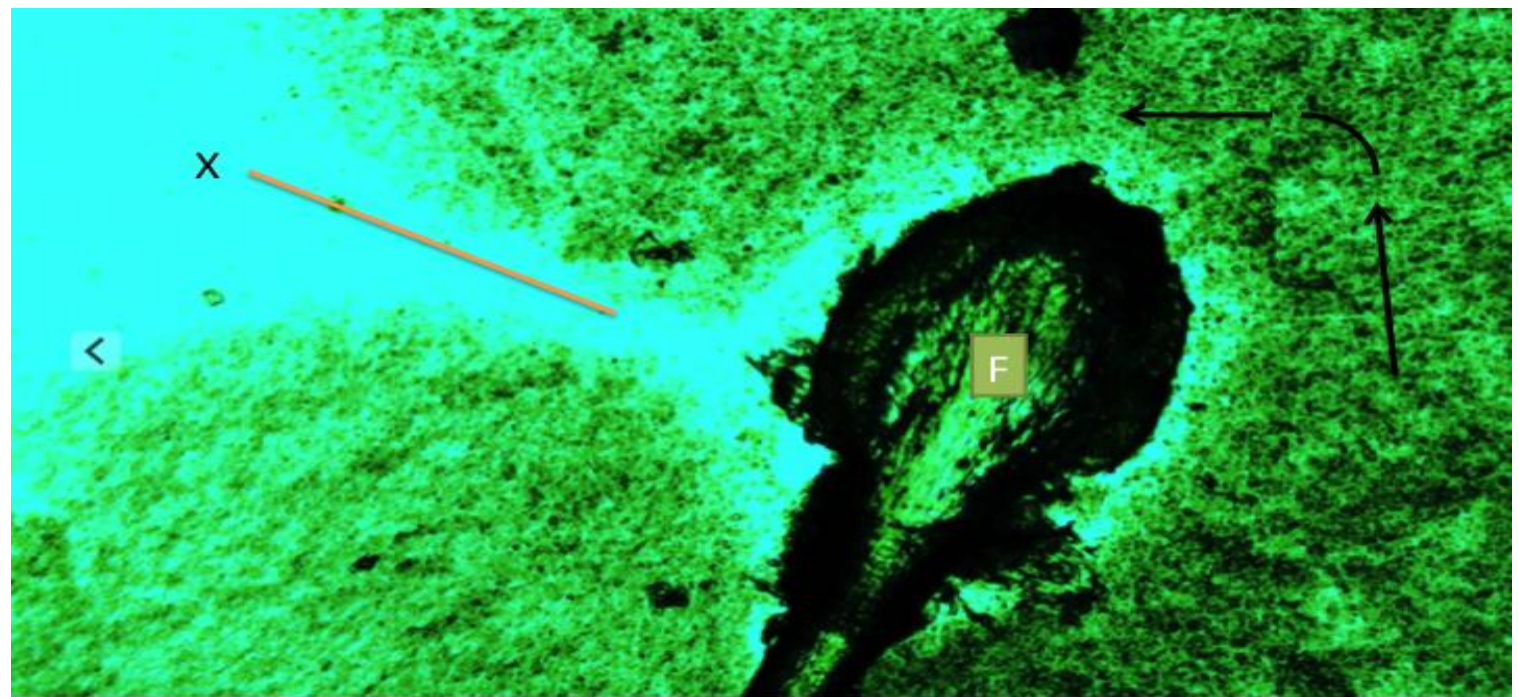

Figure 8: Selected videoframe showing: Black arrows= (right corner) flow direction of particles circulating around a hair follicle. $\mathrm{X}=$ The motion stops where the light ray displaces the particles. This preparation was a SSP PBS Fe2 2K

This is an example or the Shepherds Hook Effect. Link to video recording: http://www.jnsci.org/files/video/e55/S1.htm

Image reproduced from:

Embi AA, Jacobson JI, Sahoo K, Scherlag BJ (2015) Demonstration of Inherent Electromagnetic Energy Emanating from Isolated Human Hairs. Journal of Nature and Science, 1(3):e55.

Light Ray stopping particles progression

Unseen energy stopping crystals accretion

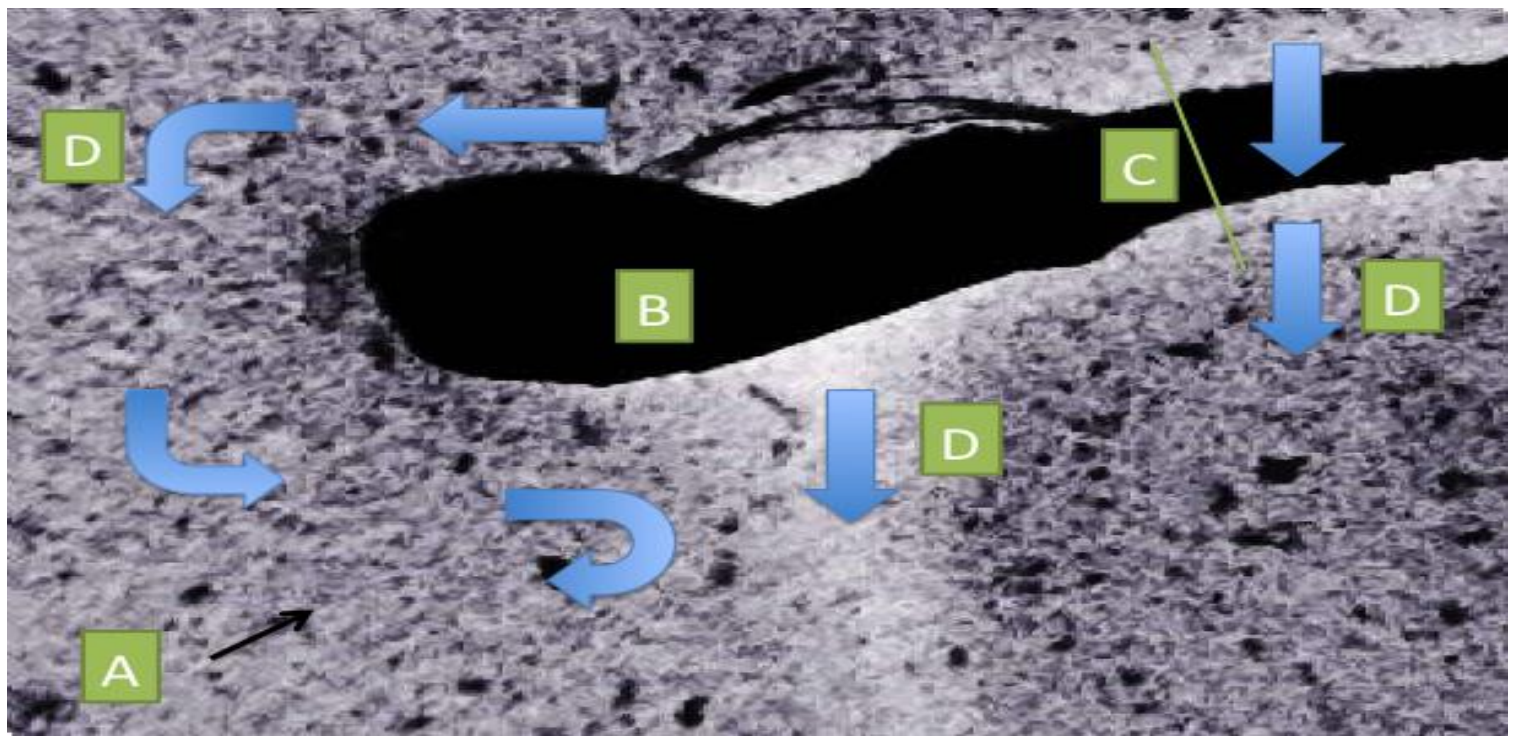

Figure 2: Initial picture taken of a large rat follicle immersed in a PBS Fe 2000 sandwich (SDW)

A) Aggregated iron nanoparticles B) Follicle C) Delineates area which is anatomically described in vivo to be under the skin ( $\mathrm{b}$ to $\mathrm{c}$ ) 
D) Iron particles movement direction. Notice the blue arrows showing the line of magnetic forces seen as a counter-clockwise rotation of the iron particles around the follicle and bulb. Also notice the seen electromagnetic radiation expressed as a light beam (D) emitted by the whisker. The magnetic counter-clockwise magnetic forces can not penetrate the light photons white trail. Also seen are the vertical motion of particles moving within the light beam, as well as across the whisker's shaft.

Embi AA, Jacobson JI, Sahoo K, Scherlag BJ (2015) Demonstration of Electromagnetic Energy Emanating from Isolated Rodent Whiskers and the Response to Intermittent Vibrations. Journal of Nature and Science, 1(3): e52.

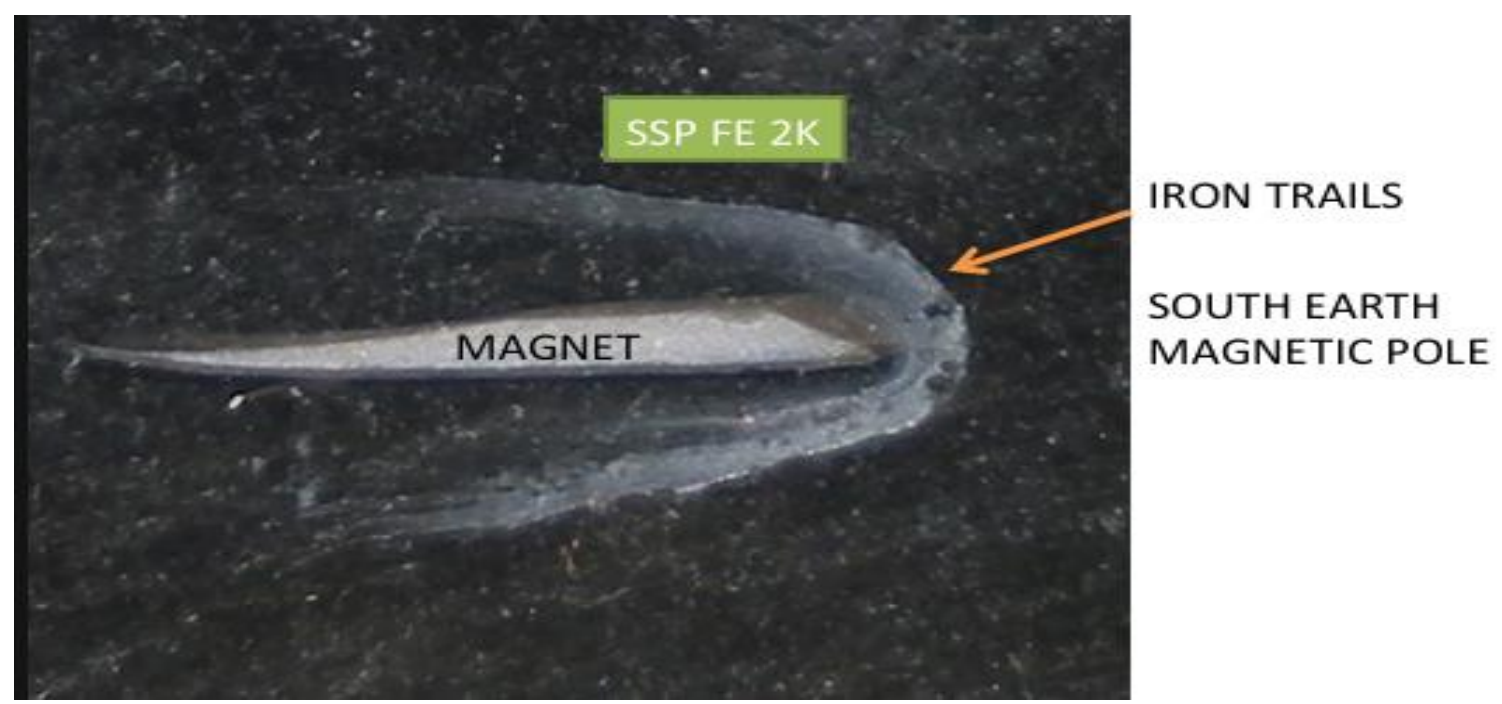

Figure 9: Showing an inanimate magnet fragment immersed in SSP Fe2K solution after evaporation. This technique only show aggregated iron particles. The south pole-oriented aggregation is a reflection of the earth terrestrial magnetism. Notice the lack of a "A Shephers Hook Pattern" since the magnet fragment is an inanimate object

\section{Discussion}

Hypothesized is that electromagnetic radiation in the form of BMFs are emitted by living tissues and move at variable speeds away from their sources. In the vicinity of these radiating charges, fine iron sized paramagnetic iron particles can be attracted and aggregate to form images stained by Prussian Blue.

In the shaft of human hairs or rodent vibrissa whiskers the BMFs are present in the external cuticles. These imbricate pattern cuticles also emit BMFs on one side of the shaft only up to and around the follicle. The contralateral area of the hair or whiskers does not display energy as seen in the opposite side. This phenomenon creates images similar to a shepherds hook best seen in (Fig 3).

\section{The Photoelectric Effect}

The etiology of this phenomenon remains undetermined; although theorized based on the images presented, that both the human follicle and the mouse whiskers emit light rays that impede the iron 
particles from completely engulfing the follicle. In other words light photons displace electrons causing the displacement of the iron laden ferrocyanides crystals.

\section{Electrons displaced from hair follicles by Laser Light}

The emission of energy in the form of light rays or flashes (by both human follicles and rodent vibrissa whiskers) are seen displacing ferrocyanide iron laden particles. This pattern of stopping crystals surrounding the follicle is only seen in living tissue. Inanimate magnet fragments fail to show this phenomenon (Fig 9). I will conclude by showing an example of the ample electrons reservoir of a hair follicle (Fig 10 below). Further research is warranted.

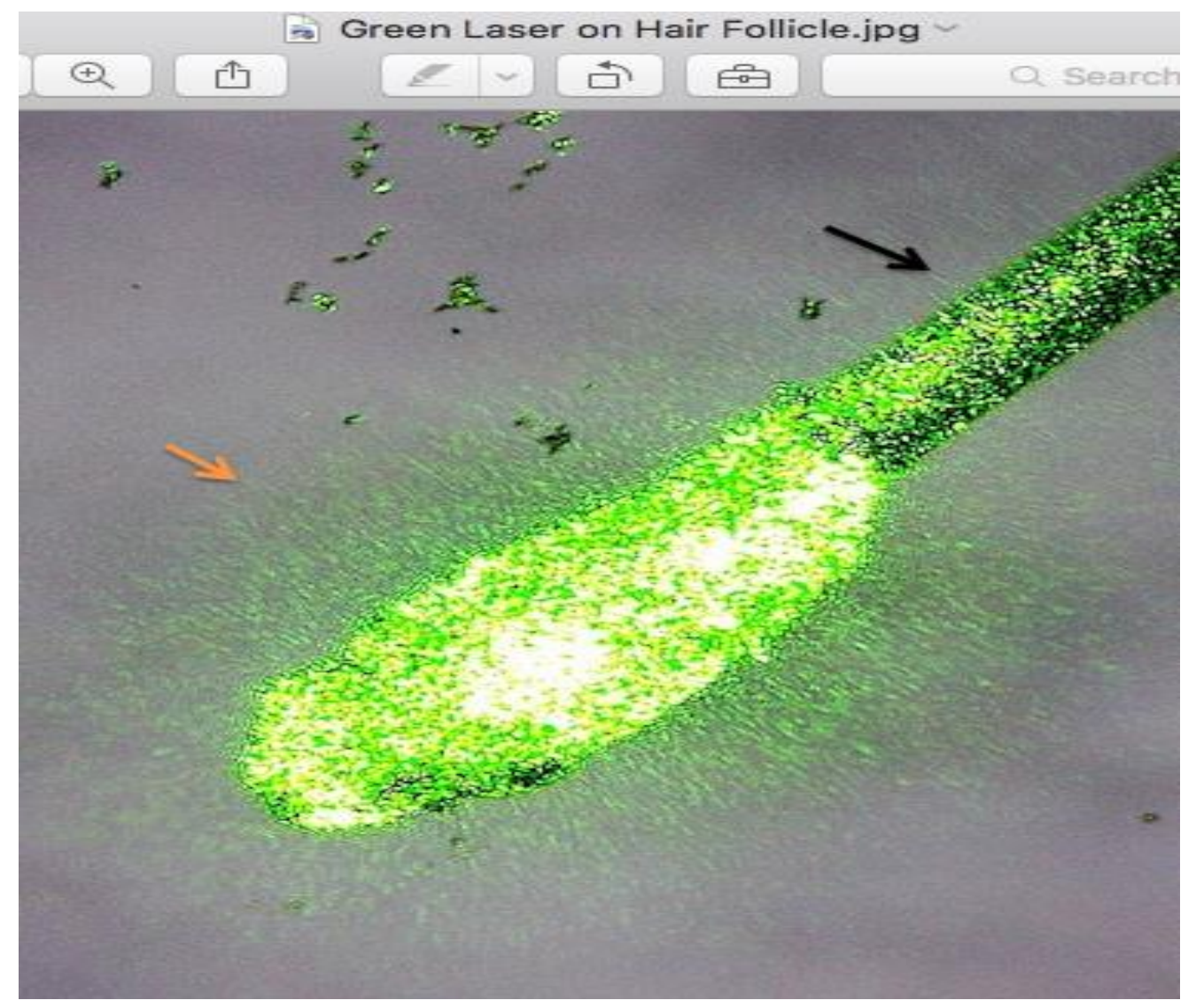

Figure 10: Green Laser light on follicle- Red Arrow: Notice spicules that are electron trails leaving the follicle, thus displacing surrounding particles. This image fits the definition of The Photoelectric Effect (light displacing electrons). Black Arrow= Pointing at hair shaft also emitting electrons

\section{References}

[1] Baule G.M, McFee R. 1963. Detection of the magnetic field of the heart. American Heart Journal ;66: 95-96 PBMID: 14045992.

[2] Cohen D. 1972. Magnetoencephalography: Detection of the Brain's electrical activity with a superconducting magnetometer. Science; 175: 664-666 PMID: 5009769.

[3] Cohen D, Kaufman LA. 1975. Magnetic determination of the relationship between the ST segment shift and the injury current produced by coronary occlusion. Circ Res;36: 414-424 PMID: 1111998. 
[4] Cohen D, Palti Y, Cuffin BN, Schmid SJ. 1980. Magnetic fields produced by steady currents in the body. Proc. Natl. Acad. Sci. USA; 77(3): 1447-1451.

[5] Demonstration of Inherent Electromagnetic Energy Emanating from Isolated Human Hairs. Abraham A. Embi, Jerry I. Jacobson, Kaustuv Sahoo, Benjamin J. Scherlag Journal of Nature and Science, 1(3): e55, 2015.

[6] Benjamin J. Scherlag, Kaustuv Sahoo, Abraham A. Embi A Novel and Simplified Method for Imaging the Electromagnetic Energy in Plant and Animal Tissues. Journal of Nanoscience and Nanoengineering Vol. 2, No. 1, 2016, pp. 6-9

[7] Embi, AA, Scherlag BJ. 2016. Demonstration of Human Hair Follicle Biomagnetic Penetration Through Glass Barriers. International Journal of materials Chemistry and Physics. Vol 2, No 2 pp: 71-74

[8] Embi AA, Jacobson JI, Sahoo K, Scherlag BJ. 2015. Demonstration of Electromagnetic Energy Emanating from Isolated Rodent Whiskers and the Response to Intermittent Vibrations. Journal of Nature and Science, 1(3): e52.

[9] Embi AA. Similarity in Bioelectromagnetic Fields Emitted by Hairs of the Mosquito Larva (Culex quinquefasciatus) and Humans. 2016. J Nat Sci, 2(11): e250.

\footnotetext{
*Corresponding author.

E-mail address: embi21@ att.net
} 